\title{
Isolated quasi-axisymmetric sunspots
}

\author{
Serge Koutchmy and Vincent Le Piouffle \\ Institut d'Astrophysique de Paris, CNRS, UPMC, 98 Bis Bd Arago, F-75014 Paris, France \\ email: koutchmy@iap.fr
}

\begin{abstract}
We briefly review the question of the origin, during a sunspot cycle, of well isolated sunspots. This includes big sunspots like the one observed in Nov. 2006. An overall axi-symmetric morphology is not perfectly observed when the morphological details of both the umbra and of the penumbra are considered. This is especially the case of umbral dots always present inside the core of a sunspot and also of penumbral filaments with non radial parts. However, the distribution of the surrounding fields, including deep layers, the occurrence of persistent coherent running penumbral waves, the magnetic moat behavior, the bright ring phenomena, etc. seem to justify a revival of the naive former but revised (converging motions are considered) Larmor model of a sunspot (as suggested by Lorrain et al. 2006). To discuss the "emergence" of single isolated sunspots from deep layers we performed a quasi-statistical analysis limited to cycle 23 . It is based on MDI data taken in the continuum, using the accompanying magnetograms to check our assertion. Surprisingly, single sunspots are definitely and preferably found to occur at low latitude and during the descending branch of the cycle. To explain our observations we speculate about the behavior of the deeply seated magnetic loop, following the original idea of $\mathrm{H}$. Alfven (with whirl rings which follow the global dipolar field when approaching the surface). It could lead to a closed loop approximately orthogonal to the local radius, similar to "smoke rings" arriving at the surface of the Sun and sometimes also called a plasmoid. The ring will only very weakly feel the destabilizing Coriolis force, when emerging at very low latitudes, which seems consistent with our observations.
\end{abstract}

Keywords. Sunspot - magnetic fields - Sun: photosphere

\section{Introduction}

Single sunspots were observed since the very beginning of visual observations of dark spots on the Sun. The father C. Scheiner and Galileo Galilee, starting in 1611, reported observations of single sunspots rotating together with the Sun, etc. This was done when obviously nobody was suspecting they are magnetic of nature and evidently, no polarity was assigned nor polarity rules were suggested to exist. Later, in his classical book printed in 1873, the father A. Secchi was still describing sunspots by selecting single sunspots (SSs) as a prototype of this complex solar feature. In the modern era, sunspots are considered as the main indicator of the solar magnetic activity and sunspot pairs of different polarities are rather preferred. In the famous Zurich classification of sunspot "groups", the SS is only at the very end of the list, making doubtful that it represents something consistent with the existing theories of the origin of sunspots. Indeed today solar physicists concentrate their attention to sunspot regions and active centers and the systematic observation of single "unipolar" and quasi-symmetric sunspots is widely neglected, although theoreticians like to consider a SS when they discuss the details of their physics, especially when the umbral core and the penumbral filaments are analyzed. In this short note there is no space to discuss the parameters of a SSs which includes an umbra, a penumbra and a surrounding moat with an opposite polarity. We just briefly mention one result of statistical significance to conclude on a possible scenario to explain this result. 


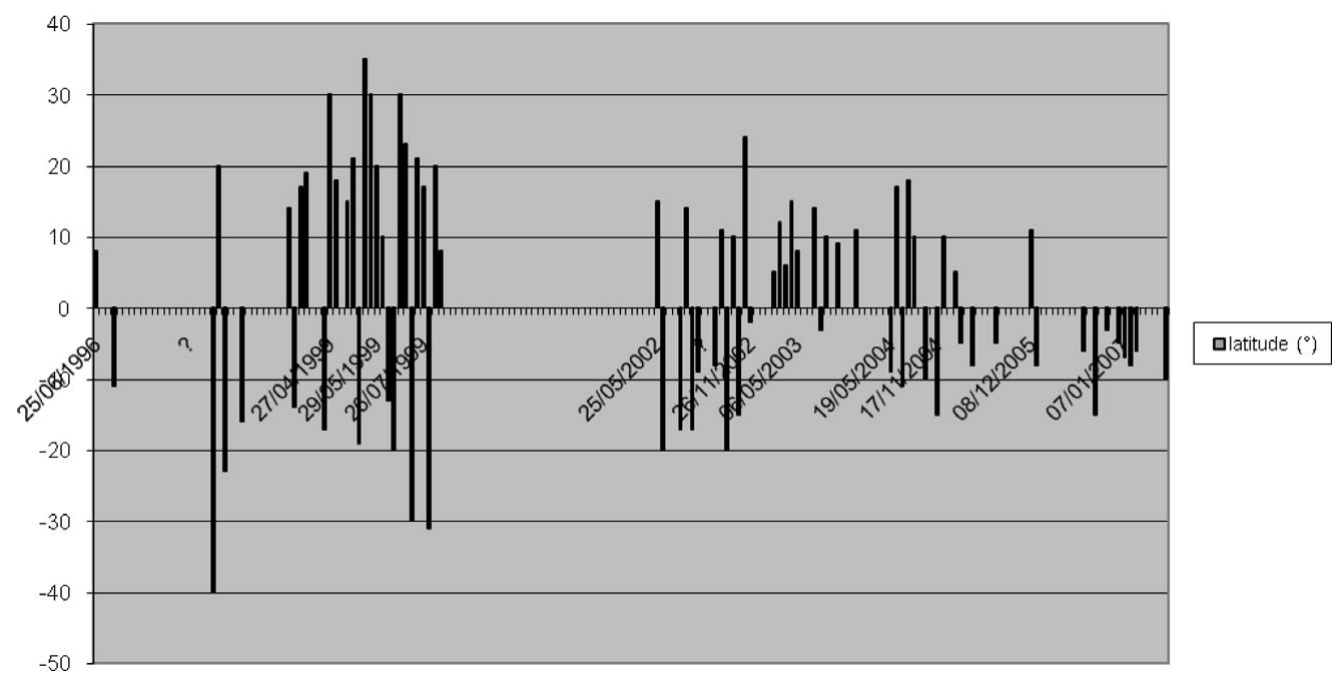

Figure 1. Dates and positions of unipolar isolated sunspots reported in this analysis of the solar cycle 23 .

\section{Analysis of Unipolar Isolated (single) Sunspots for Cycle 23}

We used the whole available data basis from MDI (SoHO) observations to look at the distribution over the disk of SSs. W-L images were examined together with the corresponding magnetograms to confirm the selection. We were interested by the frequency of appearance and the value of the latitude of the SSs. It appears that during the Years of sunspot maxima it is impossible to select the SSs, presumably because the "overlapping" effect of sunspot regions. Figure 1 gives an overview of our results shown using the usual latitude to time diagram. We further performed a finer analysis which revealed the preference of SS occurring at low latitudes. It is difficult to claim if this is due to the predominance of SSs at the end of the preceding cycle 22 or if it is a definite property of the current cycle 23 .

The occurrence of SSs at low latitude suggests that the Coriolis force could be negligible during their process of emergence. It is interesting to apply this idea to the former model suggested by Alfven (1951) when considering the path of a whirl along the global dipolar magnetic field of the Sun. Near the equator field lines are parallel to the surface and the whirl emerges almost parallel to the surface, like a smoke ring. Following the suggestion of Lorrain et al., converging motions toward the axis of the whirl will enhance any radial magnetic field which further, would form an umbra by radiative cooling, and later a penumbra. The absence of any destabilizing force could explain the occurence of really big stable SSs.

\section{References}

Alfven, H. 1951, Cosmical Electrodynamics, Oxford at the Clarendon Press, p.116 Lorrain, P. et al. 2006, Magneto-Fluid Dynamics, A\&A Library, Springer, p.205 